The aforementioned findings are seen in patients with severe burns due to direct thermal injury of RBCs circulating through the skin. Exposure to extreme heat leads to the denaturation of RBC membrane proteins, which results in hemolysis, RBC fragmentation, and vesiculation [1]. The loss of cell membrane causes the RBCs to lose their biconcavity and assume the shape of spherocytes and microspherocytes [1]. These RBC fragments and microspherocytes persist in the peripheral circulation for several days until completely removed from circulation by the reticuloendothelial system in the spleen. They are counted as platelets by aperture-based automated analyzers due to their size, leading to falsely elevated platelet counts in cases of acute burns $[1,2]$. Although reactive thrombocytosis can be seen in acute injury as recently reported by Sapanara et al. [2] in a similar burn case of a 48-year-old woman, such instances should always prompt a microscopic examination of the peripheral smear to confirm if in fact the platelet count is elevated. A manual count of platelets on peripheral smear from that patient (as in our case) revealed a normal platelet count. Such examples emphasize the importance of correlating the peripheral smear with automated $\mathrm{CBC}$ results.
Keywords: Spurious, Thrombocytosis, Burn, Platelets, Microcytosis

Anahtar Sözcükler: Yalancı, Trombositoz, Yanıklar, Trombositler, Mikrositoz

Contributors' Statement: M.F.Z. and M.S.A. both reviewed the peripheral smear and took the image. M.F.Z. wrote the first draft of the manuscript and M.S.A. revised it for intellectual content. Both authors approved the final version of the manuscript being submitted.

Conflict of Interest: The authors of this paper have no conflicts of interest, including specific financial interests, relationships, and/or affiliations relevant to the subject matter or materials included.

\section{References}

1. Lawrence $C, A$ tac $B$. Hematologic changes in massive burn injury. Crit Care Med 1992;20:1284-1288.

2. Sapanara NL, Tanev SS, Swami V. Spurious thrombocytosis in a burn patient. Lab Med 2015;35:98-100.

๑Copyright 2018 by Turkish Society of Hematology

Turkish Journal of Hematology, Published by Galenos Publishing House

\title{
A Rare Cause of Paraplegia: Myeloid Sarcoma
}

\author{
Nadir Bir Parapleji Nedeni: Myeloid Sarkom
}

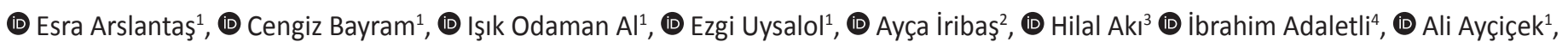
(1) Nihal Özdemir ${ }^{1}$

${ }^{1}$ University of Health Sciences, Kanuni Sultan Süleyman Traning and Research Hospital, Clinic of Pediatric Hematology and Oncology, Istanbul, Turkey

${ }^{2}$ istanbul University Institute of Oncology, Department of Radiation Oncology, Istanbul, Turkey

${ }^{3}$ istanbul University Cerrahpaşa Faculty of Medicine, Department of Pathology, İstanbul, Turkey

${ }^{4}$ istanbul University Cerrahpaşa Faculty of Medicine, Department of Radiology, Istanbul, Turkey

To the Editor,

Myeloid sarcoma (MS), also known as granulocytic sarcoma or chloroma, is a rare extramedullary tumor consisting of myeloblasts or immature myeloid cells that disrupt the normal architecture of the involved tissue and typically occurs concurrently with acute myeloid leukemia (AML) [1,2]. It can also occur in association with accelerated-phase chronic myeloid leukemia or myelodysplastic syndrome; as an extramedullary relapse of AML, including in the post-bone marrow transplant setting; and occasionally as the first presenting manifestation, even before bone marrow involvement $[3,4]$. Bone, periosteum, skin, orbit, lymph nodes, the gastrointestinal tract, and the central nervous system are the most commonly involved sites in patients presenting with MS; however, skin and orbital localizations are the most often reported sites in children [4]. Here we present a 4-year-old male patient who was referred to the pediatric hematology oncology clinic due to a thoracolumbar mass and subsequently diagnosed with MS. 


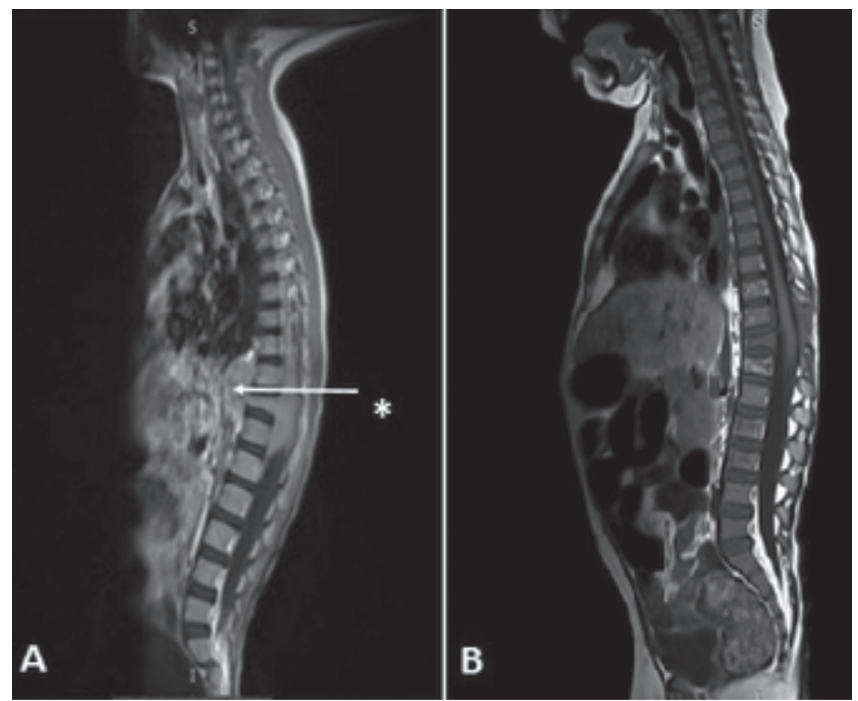

Figure 1. A) Sagittal T1-weighted MRI image showing an epidural, hypointense, craniocaudal mass of $4.5 \mathrm{~cm}$ in diameter compressing the spinal cord at the level of D 10-12; B) image of the mass 1 month before completion of acute myeloid leukemia maintenance therapy.

A 4-year-old boy was referred to the pediatric hematology oncology clinic with the complaint of hemiparesis and a subsequent thoracolumbar mass was detected by magnetic resonance imaging (MRI) (Figure 1A). On physical examination, bilateral lower extremity paralysis was noted and deep tendon reflexes were absent. Complete blood count and blood biochemical analysis were normal, and no blasts were detected on peripheral blood film. Bone marrow aspiration showed $30 \%$ blasts compatible with AML. The pathology of the mass revealed MS. After administration of radiotherapy, given at a dose of 18 Gy in 10 daily fractions in 2 weeks, and dexamethasone therapy, the patient achieved neurological improvement. He was treated with the AML-Berlin Frankfurt Münster 2012 protocol and achieved both remission and mass reduction following AML induction chemotherapy. The patient is still in remission without any residual tumor on follow-up MRI (Figure 1B).

MS may occur at any site of the body, and therefore clinical manifestations of MS exhibit diversity depending on the specific location and size, which leads to significant diagnostic challenges, in particular in patients without initial bone marrow involvement.
Incorrect diagnosis of malignant lymphoproliferative disorders, Ewing's sarcoma, thymoma, melanoma, round blue cell tumors, or poorly differentiated carcinoma has been reported at a rate of 25\%-47\% in patients subsequently diagnosed with MS. In this regard, any atypical cellular infiltrate should raise the suspicion of MS to make a correct diagnosis in a timely manner and to allow for proper management $[2,4,5]$. Diagnostic tools for the correct diagnosis of MS are also important in this context and should include MRI and/or computed tomography scan for evaluation of the size and location of the tumor and for distinguishing the tumor from other lesions, morphological and flow cytometric analysis of bone marrow and peripheral blood, or biopsy of the tumor and immunohistochemical staining in patients without bone marrow involvement [4]. Treatment of MS includes AML-based protocols and, as in our case, surgery and/or radiotherapy may be indicated for symptomatic lesions or tumors causing local organ dysfunction [5]. Considering the most common presentation sites in children with MS, which are skin and orbital localizations, the current patient is presented to highlight a rarely encountered presenting feature of MS.

Keywords: Myeloid sarcoma, Children, Paraplegia

Anahtar Sözcükler: Myeloid sarkom, Çocuk, Parapleji

Conflict of Interest: The authors of this paper have no conflicts of interest, including specific financial interests, relationships, and/or affiliations relevant to the subject matter or materials included.

\section{References}

1. Reinhardt D, Creutzig U. Isolated myelosarcoma in children-update and review. Leuk Lymphoma 2002;43:565-574.

2. Bakst RL, Tallman MS, Douer D, Yahalom J. How I treat extramedullary acute myeloid leukemia. Blood 2011;118:3785-3793.

3. Redner A, Kessel R. Acute myeloid leukemia. In: Lanzkowsky P, Lipton JM, Fish JD (eds). Lanzkowsky's Manual of Pediatric Hematology and Oncology. 6th ed. Amsterdam, Elsevier, 2016.

4. Samborska M, Derwich K, Skalska-Sadowska J, Kurzawa P, Wachowiak J. Myeloid sarcoma in children - diagnostic and therapeutic difficulties. Contemp Oncol (Pozn) 2016;20:444-448.

5. Almond LM, Charalampakis M, Ford SJ, Gourevitch D, Desai A. Myeloid sarcoma: presentation, diagnosis, and treatment. Clin Lymphoma Myeloma Leuk 2017;17:263-267. 